\title{
NONLINEAR UNSTEADY FLOW PROBLEMS BY MULTIDIMENSIONAL SINGULAR INTEGRAL REPRESENTATION ANALYSIS
}

\author{
E. G. LADOPOULOS
}

Received 10 December 2001

\begin{abstract}
A two-dimensional nonlinear aerodynamics representation analysis is proposed for the investigation of inviscid flowfields of unsteady airfoils. Such problems are reduced to the solution of a nonlinear multidimensional singular integral equation as the source and vortex strength distributions are dependent on the history of these distributions on the NACA airfoil surface. A turbulent boundary layer model is further investigated, based on the formulation of the unsteady behaviour of the momentum integral equation. An application is finally given to the determination of the velocity and pressure coefficient field around an aircraft by assuming linear vortex distribution.
\end{abstract}

2000 Mathematics Subject Classification: 65L10, 65R20.

1. Introduction. Over the last years, an increasing interest has been concentrated on the study of nonlinear multidimensional singular integral equations because of their application to the solution of modern and complicated problems of solid and fluid mechanics theory. Such problems are solved by computational methods as closed-form solutions are not possible to be determined. The algorithms which are used for the numerical evaluation of the nonlinear singular integral equations consist with the latest high technology for the solution of modern problems of solid mechanics, fluid mechanics, and aerodynamics.

The design of the new generation aircrafts with very high speeds has recently become too important, driven by the needs of aircraft powerplant and turbine designers. The target of the aeronautical industries all over the world is to achieve a competitive technological advantage in certain strategic areas of new and rapidly developing advanced technologies. Such a considerably market share includes the design of new generation of large aircrafts with very high speeds. Furthermore, the new technology aerodynamic problems are reduced to the solution of nonlinear multidimensional singular integral equations, used for the determination of the velocity and pressure coefficient field around the NACA airfoils.

Hess and Smith [6] were the first scientists who investigated aerodynamic panel methods for studying airfoils with zero lift. In their study, they modeled 
the airfoil with either distributed potential source panels for nonlifting flows, or vortex panels for flow with lift. The above method was further extended by Djojodihardjo and Widnall [4], Robert and Saaris [22], Summa [26], Bristow [1], Bristow and Hawk [2], and Lewis [18], as they studied three-dimensional steady and unsteady flows by combining source and vortex singularities. Furthermore, Sarpkaya and Schoaff [24] extended the unsteady panel methods to the modeling of separated wakes using discrete vortices.

Beyond the above, some potential flow models were investigated by Ham [5], Deffenbaugh and Marshall [3], Kiya and Arie [9], and Sarpkaya and Kline [23], and the separating boundary layers were represented by an array of discrete vortices, emanating from a known separation point location on the airfoil surface.

During the last years, several other scientists made extensive calculations by using unsteady turbulent boundary layer methods. Among them Singleton and Nash [25], Nash et al. [21], Lyrio et al. [19], McCroskey and Pucci [20], and Kim et al. [8].

Recently, nonlinear singular integral equation methods were proposed by Ladopoulos [10, 11, 12, 13, 14] for the solution of fluid mechanics problems and by Ladopoulos and Zisis [15, 16] for two-dimensional fluid mechanics problems applied to turbomachines.

In the present paper, the aerodynamic problem of the unsteady flow of a two-dimensional NACA airfoil which is moving by a velocity $\mathbf{U}_{A}$ is reduced to the solution of a nonlinear multidimensional singular integral equation. This nonlinearity is valid as the source and vortex strength distributions are dependent on the history of the vorticity and source distributions on the NACA airfoil surface. Furthermore, a turbulent boundary layer analysis is investigated, based on the formulation of the unsteady behaviour of the momentum integral equation.

An application is finally given to the determination of the velocity and pressure coefficient field around an aircraft by assuming linear vortex distribution.

2. Nonlinear two-dimensional aerodynamics analysis. Consider the motion of a two-dimensional airfoil through a homogeneous and inviscid fluid (Figure 2.1).

The airfoil plus the wake comprise a complete lifting system which makes the motion irrotational for all time $[10,11,12,13,14]$. Because of this irrotationality, the local fluid velocity $\mathbf{U}$ is valid

$$
\nabla \times \mathbf{U}=0
$$

Let $H$ be the total velocity potential. Then, by replacing the fluid velocity $\mathbf{U}$ with the total velocity potential, one obtains

$$
\mathbf{U}=\nabla H
$$




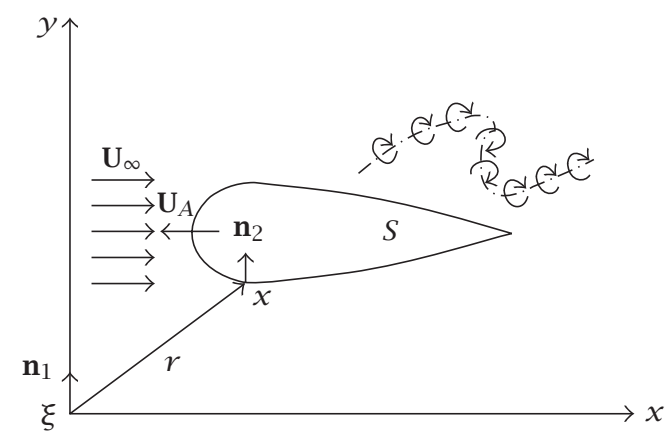

FIGURE 2.1. A two-dimensional airfoil of surface $S$ in an homogeneous and inviscid fluid.

By considering $\mathbf{U}_{\infty}$ the outward velocity (Figure 2.1 ) and $h$ the velocity potential due to the presence of the airfoil, (2.2) can be further written as

$$
\mathbf{U}=\mathbf{U}_{\infty}+\nabla h .
$$

Beyond the above, the use of Green's theorem [17] results in the following relation for the velocity potential $h(\mathbf{x}, t)$, with $t$ the time, at any point $\mathbf{x}$ in a continuous acyclic irrotational flow:

$$
h(\mathbf{x}, t)=\frac{-1}{2 \pi} \int_{S} \frac{\omega[\xi, t, h]}{r} d S+\frac{1}{2 \pi} \int_{S+W} \delta[\xi, t, h] \frac{\partial}{\partial n_{1}}\left(\frac{1}{r}\right) d S
$$

in which $S$ denotes the surface of the airfoil (Figure 2.1), $W$ the surface of the wake, $\mathbf{n}_{1}$ the surface normal at the source point $\xi$ (Figure 2.1), $\omega[\xi, t, h]$ the source strength distribution, $\delta[\xi, t, h]$ the vortex strength distribution, and $r$ the distance equal to

$$
r=|\mathbf{x}-\xi|
$$

Moreover, (2.4) takes the following form, which denotes a two-dimensional nonlinear singular integral equation:

$$
h(\mathbf{x}, t)=\frac{-1}{2 \pi} \int_{S} \frac{\omega[\xi, t, h]}{r} d S+\frac{1}{2 \pi} \int_{S+W} \frac{\delta[\xi, t, h]}{r^{2}} d S .
$$

The kinematical surface tangency condition on the surface of the airfoil is equal to (see [7])

$$
\frac{1}{|\nabla S(\mathbf{x}, t)|} \frac{\partial S(\mathbf{x}, t)}{\partial t}+\frac{\partial f}{\partial n_{2}}+\mathbf{U}_{\infty} \cdot \mathbf{n}_{2}=0
$$

where $\mathbf{n}_{2}$ denotes the surface normal at the field point $\mathbf{x}$ (Figure 2.1). 
For a body fixed coordinate system, this condition takes the form

$$
\frac{1}{|\nabla S(\mathbf{x}, t)|} \frac{\partial S(\mathbf{x}, t)}{\partial t}=-\left(\mathbf{U}_{A}+\boldsymbol{\omega}_{A} \times \mathbf{x}\right) \cdot \mathbf{n}_{2}
$$

in which $\mathbf{U}_{A}$ denotes the airfoil translation velocity and $\boldsymbol{\omega}_{A}$ the airfoil angular rotation.

From (2.7) and (2.8), it follows that

$$
\frac{\partial h}{\partial n_{2}}+\left(\mathbf{U}_{\infty}-\mathbf{U}_{A}-\boldsymbol{\omega}_{A} \times \mathbf{x}\right) \cdot \mathbf{n}_{2}=0 .
$$

Hence, by inserting (2.9) into (2.6), a two-dimensional nonlinear singular integral equation follows:

$$
\begin{gathered}
\frac{1}{2 \pi} \int_{S} \omega[\xi, t, h] \frac{\partial}{\partial n_{2}}\left(\frac{1}{r}\right) d S+\frac{1}{2 \pi} \int_{S+W} \delta[\xi, t, h] \frac{\partial}{\partial n_{2}}\left(\frac{1}{r^{2}}\right) d S \\
=-\left(\mathbf{U}_{\infty}-\mathbf{U}_{A}-\boldsymbol{\omega}_{A} \times \mathbf{x}\right) \cdot \mathbf{n}_{2} .
\end{gathered}
$$

The nonlinear singular integral equation (2.10) can be further written as

$$
\frac{1}{2 \pi} \int_{S} \frac{\omega[\xi, t, h]}{r^{2}} d S+\frac{1}{\pi} \int_{S+W} \frac{\delta[\xi, t, h]}{r^{3}} d S=\left(\mathbf{U}_{\infty}-\mathbf{U}_{A}-\omega_{A} \times \mathbf{x}\right) \cdot \mathbf{n}_{2} .
$$

Finally, by solving the nonlinear integral equation (2.11) with the corresponding boundary conditions, the velocity at any field point will be determined through (2.7).

3. Nonlinear airloads analysis. By using the unsteady Bernoulli equation, valid at any point in an irrotational ideal flow, the pressure distribution on the airfoil can be determined as

$$
P=P_{\infty}-\rho\left[\frac{\partial H}{\partial t}+\frac{1}{2}(\nabla H)^{2}\right]
$$

where $\rho$ is the fluid density.

By using the derivation of the previous section, (3.1) becomes

$$
P=P_{\infty}-\rho\left[\frac{\partial h}{\partial t}+\left(\mathbf{U}_{\infty}-\mathbf{U}_{A}-\boldsymbol{\omega}_{A} \times \mathbf{x}\right) \cdot \nabla h+\frac{1}{2}(\nabla h)^{2}\right] .
$$

Beyond the above, (3.2) takes the form

$$
\begin{aligned}
P=P_{\infty}-\rho[ & \frac{\partial H}{\partial t}+\left(\mathbf{U}_{\infty}-\mathbf{U}_{A}-\boldsymbol{\omega}_{A} \times \mathbf{x}\right) \cdot \nabla_{S} H \\
& \left.+\frac{\partial H}{\partial n_{1}}\left(\mathbf{U}_{\infty}-\mathbf{U}_{A}-\boldsymbol{\omega}_{A} \times \mathbf{x}\right) \cdot \mathbf{n}_{1}+\frac{1}{2}\left(\nabla_{S} H\right)^{2}+\frac{1}{2}\left(\frac{\partial H}{\partial n_{1}}\right)^{2}\right]
\end{aligned}
$$


if we replace $\nabla h$ by the surface gradient $\nabla_{S} h$ :

$$
\nabla h=\nabla_{S} h+\frac{\partial h}{\partial n_{1}} \varepsilon_{n_{1}}
$$

Because of (2.11), (3.3) takes the form

$$
\begin{aligned}
P=P_{\infty}-\rho[ & \frac{\partial H}{\partial t}+\left(\mathbf{U}_{\infty}-\mathbf{U}_{A}-\boldsymbol{\omega}_{A} \times \mathbf{x}\right) \cdot \nabla_{S} H \\
& \left.-\frac{1}{2}\left\{\left(\mathbf{U}_{\infty}-\mathbf{U}_{A}-\boldsymbol{\omega}_{A} \times \mathbf{x}\right) \cdot \mathbf{n}_{1}\right\}^{2}+\frac{1}{2}\left(\nabla_{S} H\right)^{2}\right],
\end{aligned}
$$

which will be used for the computations.

The basic object of the present paper is to develop a general nonlinear model for the determination of the velocity field around a NACA airfoil in a twodimensional unsteady flow. This problem was reduced to the solution of a two-dimensional nonlinear singular integral equation, while the above form of nonlinearity was obtained because of the special kind of the general type of the source and vortex strength distribution.

4. Turbulent boundary layer models. The boundary layer model which would be ideal for the aerodynamic behaviour of the airfoil should be able to predict both laminar and turbulent parts of the flow as well as the transition region between them. There are several boundary layer models which can be used, like the finite difference, finite element, or integral models.

In this paper, the proposed turbulent boundary layer analysis will be based on the formulation of the unsteady behaviour of the momentum integral equation. Hence, the unsteady momentum integral equation valid for both laminar and turbulent flows is equal to (see Figure 4.1)

$$
\frac{1}{u_{B}^{2}} \frac{\partial}{\partial t}\left(u_{B} \delta\right)+\frac{\partial d}{\partial S}+\frac{1}{u_{B}} \frac{\partial u_{B}}{\partial S}(2 d+S)=\frac{c_{F}}{2}
$$

where $u_{B}$ denotes the boundary layer edge velocity, $t$ the time, $\delta$ the displacement thickness, $d$ the momentum thickness, $S$ the surface distance, and $c_{F}$ is the friction factor.

For the laminar layer, a pressure gradient parameter $\lambda$ is written as

$$
\lambda=\frac{d}{u_{B}} R_{d}\left(\frac{\partial u_{B}}{\partial S}+\frac{1}{u_{B}} \frac{\partial u_{B}}{\partial t}\right)
$$

in which $R_{d}$ is the Reynolds number based on $u_{B}$ and $d$. 


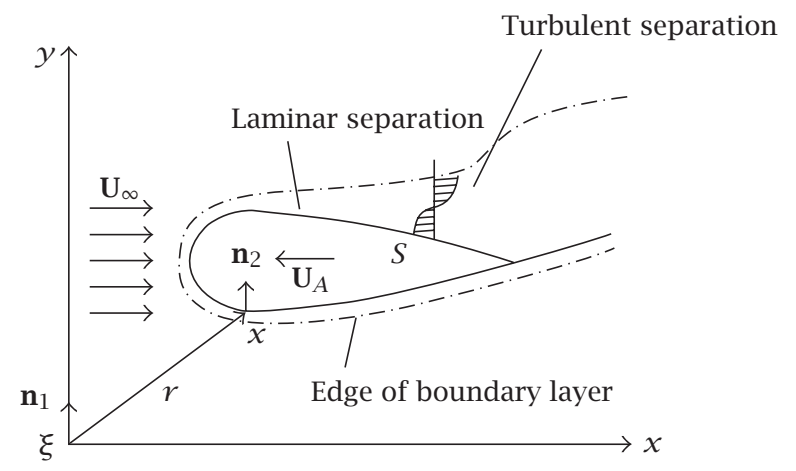

FIGURE 4.1. Laminar and turbulent boundary layer model for aerodynamics.

Beyond the above, a solution for the laminar formulation is obtained, by considering relations between the parameters $c_{F} / 2, d$, and $\delta$. For wedge flow solutions, one has

$$
\begin{aligned}
\frac{c_{F}}{2} & =\frac{1.91-4.13 L}{R_{\delta}}, \\
N & =(0.68-0.922 L)^{-1}, \\
L & =0.325-0.13 \lambda N^{2},
\end{aligned}
$$

where $N$ denotes the shape parameter, $L$ the blockage factor $\delta / \delta_{B}$ with $\delta_{B}$ the boundary layer thickness, and $R_{\delta}$ the Reynolds number based on $u_{\delta}$ and $\delta$.

For the turbulent layer model, the following equation is valid:

$$
\frac{1}{u_{B}} \frac{\partial}{\partial S}\left[u_{B}\left(\delta_{B}-\delta\right)\right]=T
$$

and the function $T$ is obtained by the relations

$$
\begin{aligned}
\frac{d T}{d S} & =0.025\left(T_{B}-T\right) \delta_{B}, \\
T_{B} & =4.24 K_{B}\left(\frac{L}{1-L}\right)^{0.916}, \\
K_{B} & =0.013+0.0038 e^{-\beta / 15}, \\
\beta & =\frac{\delta}{\tau_{w}} \frac{d P}{d x},
\end{aligned}
$$

with $\tau_{w}$ the wall shear stress and $d P / d x$ the streamwise pressure gradient. 
Shape factor relationships are obtained by the following formulas:

$$
\begin{aligned}
\frac{u}{u_{B}} & =1+\mu \ln \left(\frac{y}{\delta_{B}}\right)-\varphi \cos ^{2}\left(\frac{\pi y}{2 \delta_{B}}\right), \\
\mu & =\frac{1}{0.41}\left(\operatorname{sgn} \frac{c_{F}}{2}\right)\left(\frac{c_{F}}{2}\right)^{1 / 2}, \\
\varphi & =2(1-\mu), \\
\frac{c_{F}}{2} & =\frac{\tau_{w}}{\rho u_{B}^{2}},
\end{aligned}
$$

where $u$ is the velocity in the boundary layer at a distance $y$ from the wall and $\rho$ the fluid density.

Finally, the skin friction law is

$$
\frac{C_{F}}{2}=0.051|1-2 L|^{1.732}\left(\frac{R_{\delta}}{L}\right)^{-0.268} \operatorname{sgn}(1-2 L) .
$$

5. Velocity and vortex coefficient field for linear vortex distribution (airfoil with velocity). Consider the special case of a linear vortex distribution $\delta$. In this case the general nonlinear problem presented in previous paragraphs will be much more simplified and will be solved as a linear problem. The geometrical representation of this problem is shown in Figure 5.1.

For a linear vortex distribution $\delta$, the fluid velocity $\mathbf{U}$ is given by the relation

$$
\mathbf{U}=\int_{-A / 2}^{A / 2} \frac{\delta d r}{2 \pi r}(-\sin \varphi \mathbf{i}+\cos \varphi \mathbf{j})
$$

where $A$ denotes the separating wake (Figure 5.1) and $\mathbf{i}, \mathbf{j}$ the unit vectors on the $x$ - and $y$-axis, respectively.

Therefore, the fluid velocity $\mathbf{U}$ will be computed through the following relations, for the cases $y_{P} \neq 0$ and $y_{P}=0$ :

$$
\mathbf{U}=\left\{\begin{array}{cc}
\frac{a}{2 \pi}\left[x_{P}\left(\varphi_{1}-\varphi_{2}\right)+y_{P} \ln \left|\frac{r_{1}}{r_{2}}\right|\right] \mathbf{i} & \\
+\frac{a}{2 \pi}\left[x_{P} \ln \left|\frac{r_{1}}{r_{2}}\right|-A-y_{P}\left(\varphi_{1}-\varphi_{2}\right)\right] \mathbf{j}, & y_{P} \neq 0, \\
\frac{a}{2 \pi}\left[x_{P} \ln \left|\frac{r_{1}}{r_{2}}\right|-A\right] \mathbf{j}, & y_{P}=0,
\end{array}\right.
$$

where $a$ is the angle of attack. 


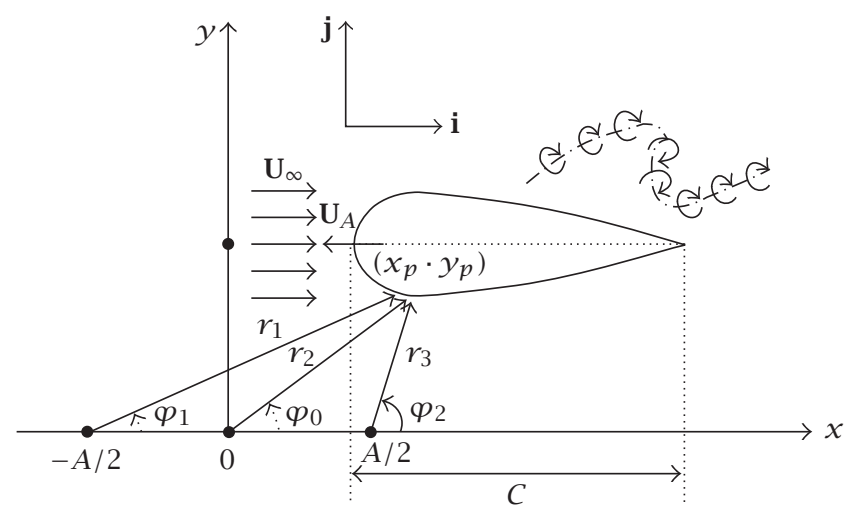

FIGURE 5.1. Coordinate system for 2D airfoil of an aircraft.

Beyond the above, consider the pressure coefficient

$$
C_{P}=\frac{P-P_{\infty}}{(1 / 2) \rho\left(U_{\infty}-U_{A}\right)^{2}},
$$

in which $P_{\infty}$ denotes the stream pressure and $\rho$ the fluid density.

By further using the unsteady Bernoulli equation, the pressure coefficient will be simplified as follows

$$
C_{P}=-\frac{U^{2}}{\left(U_{\infty}-U_{A}\right)^{2}},
$$

which will be used for the computations.

6. Aircraft application. The previous mentioned theory of 2D unsteady inviscid flowfields will be applied for the computation of the velocity and pressure coefficient field around an aircraft. The big evolution of the jet engine and the high performance axial-flow compressor have considerably increased the possibilities of turbomachines applied in aircrafts. The further application of the new generation turbojet engines makes the design of very fast large aircrafts possible.

In the present application, the length of the aircraft under consideration is $c=50.0 \mathrm{~m}$ and the airfoil section is NACA 0021 (Figure 5.1).

Also, linear vortex distribution was supposed and hence the velocity field on the boundary around the airfoil was computed by (5.2). Moreover, the pressure coefficients $C_{P}$ were calculated by (5.4) for several aircraft velocities $U_{A}$ and wind velocity $U_{\infty}=15 \mathrm{~m} / \mathrm{s}$ and for angle of attack $a=30^{\circ}$.

Hence, Figures 6.1, 6.3, 6.5, and 6.7 show the pressure distribution on the aircraft considered, for aircraft speed $U_{A}=1,2,3,4$ Mach, respectively ( 1 Mach = $332 \mathrm{~m} / \mathrm{s}$ ). Also, Figures 6.2, 6.4, 6.6, and 6.8 show the same pressure distribution on the airfoil in three-dimensional form. 


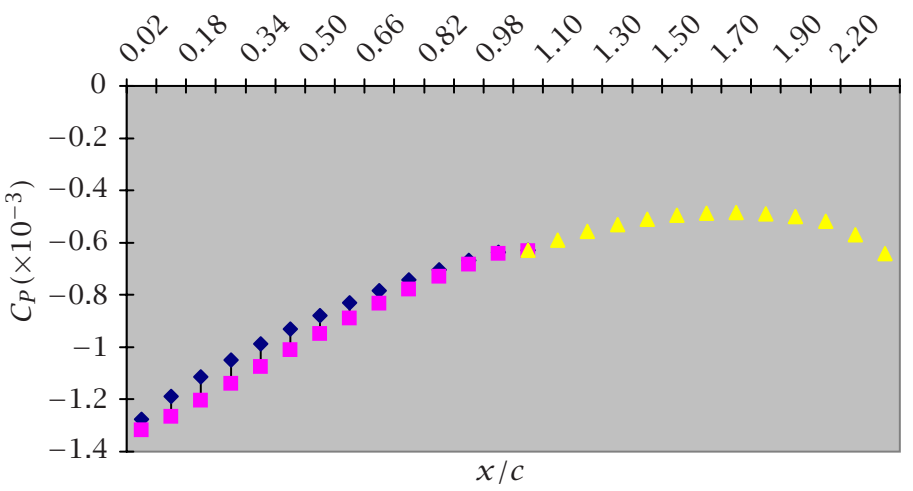

- Up-boundary points

- Down-boundary points

$\Delta$ Out points

FIGURE 6.1. Pressure distribution around the aircraft of Figure 5.1, for linear vortex distribution and speed 1 Mach.

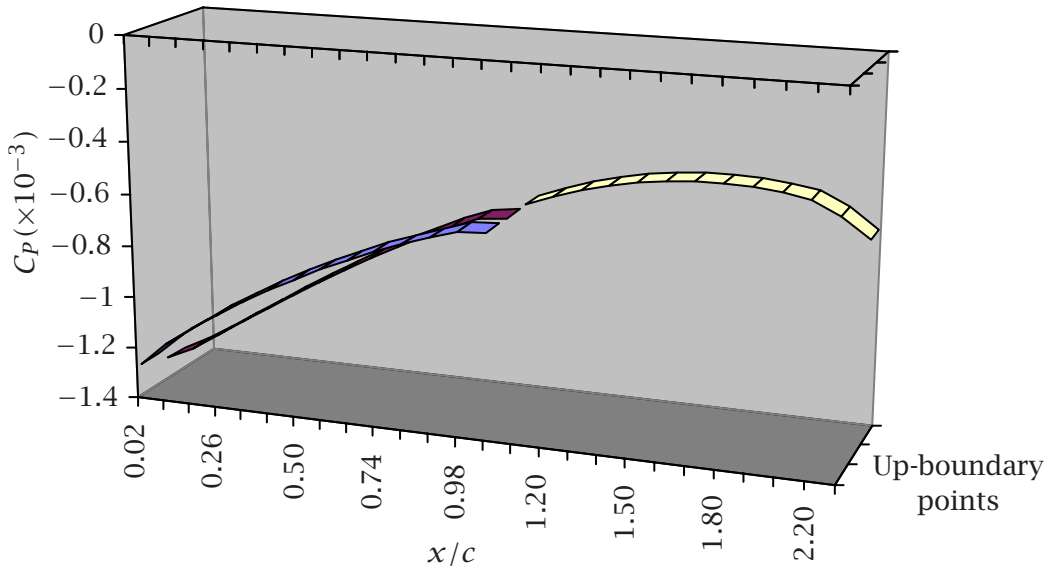

$\square$ Up-boundary points

$\square$ Down-boundary points

$\square$ Out points

FIGURE 6.2. Pressure distribution (3D form) around the aircraft of Figure 5.1, for linear vortex distribution and speed 1 Mach. 


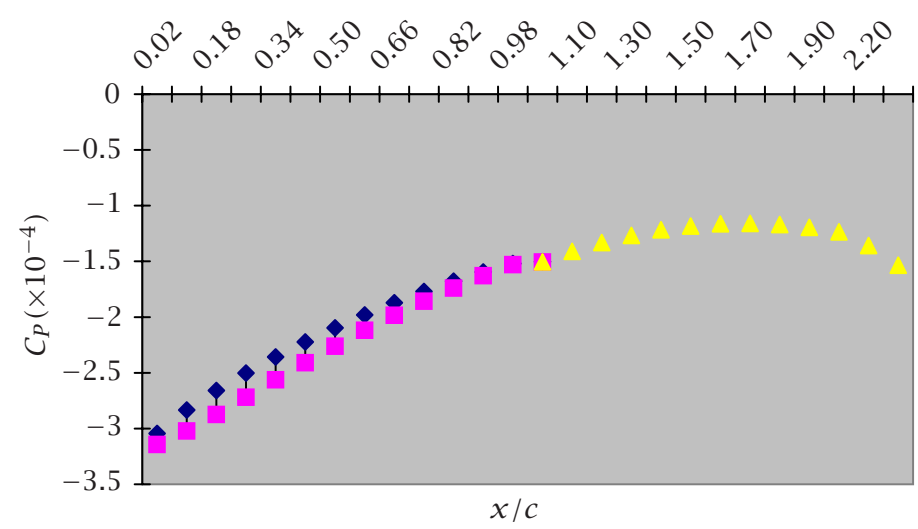

- Up-boundary points

- Down-boundary points

$\triangle$ Out points

FIGURE 6.3. Pressure distribution around the aircraft of Figure 5.1, for linear vortex distribution and speed 2 Mach.

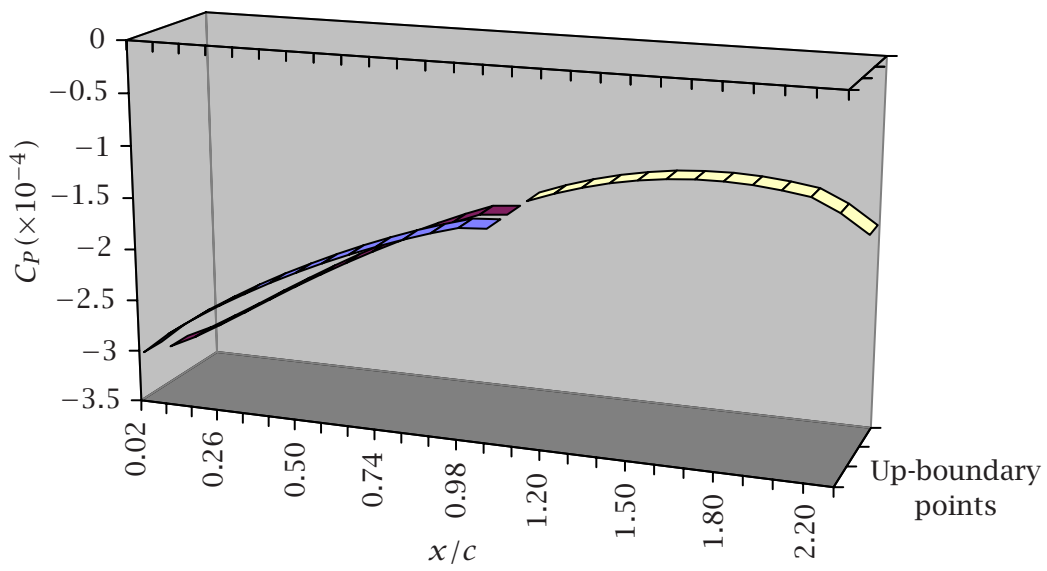

Up-boundary points

$\square$ Down-boundary points

$\square$ out points

FIGURE 6.4. Pressure distribution (3D form) around the aircraft of Figure 5.1, for linear vortex distribution and speed 2 Mach. 


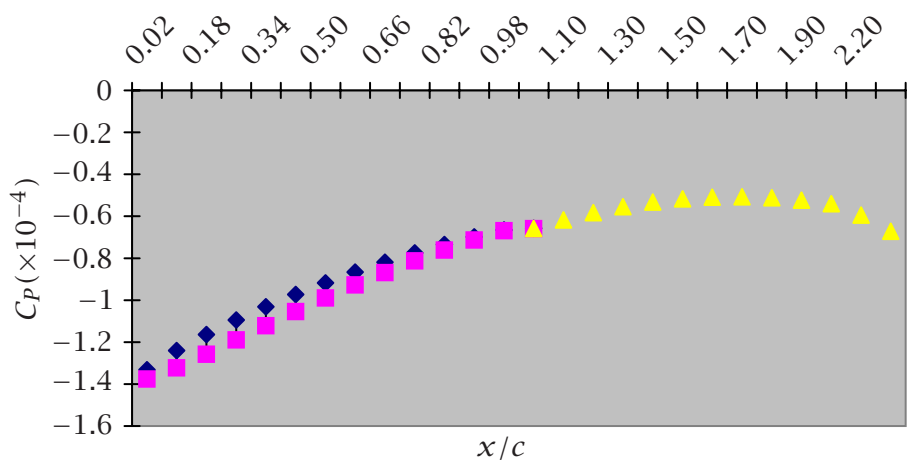

- Up-boundary points

Down-boundary points

Out points

FIGURE 6.5. Pressure distribution around the aircraft of Figure 5.1, for linear vortex distribution and speed 3 Mach.

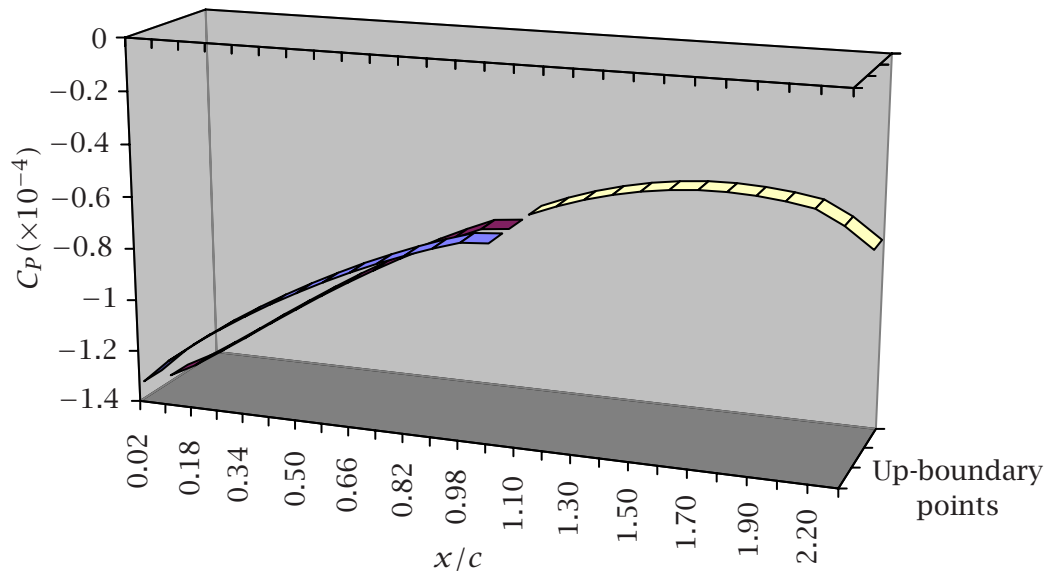

$\square$ Up-boundary points

$\square$ Down-boundary points

$\square$ Out points

FIGURE 6.6. Pressure distribution (3D form) around the aircraft of Figure 5.1, for linear vortex distribution and speed 3 Mach. 


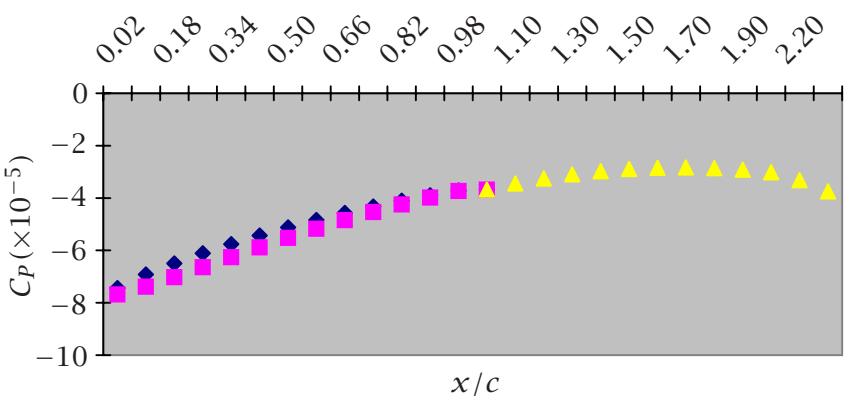

- Up-boundary points

- Down-boundary points

Out points

FIGURE 6.7. Pressure distribution around the aircraft of Figure 5.1, for linear vortex distribution and speed 4 Mach.

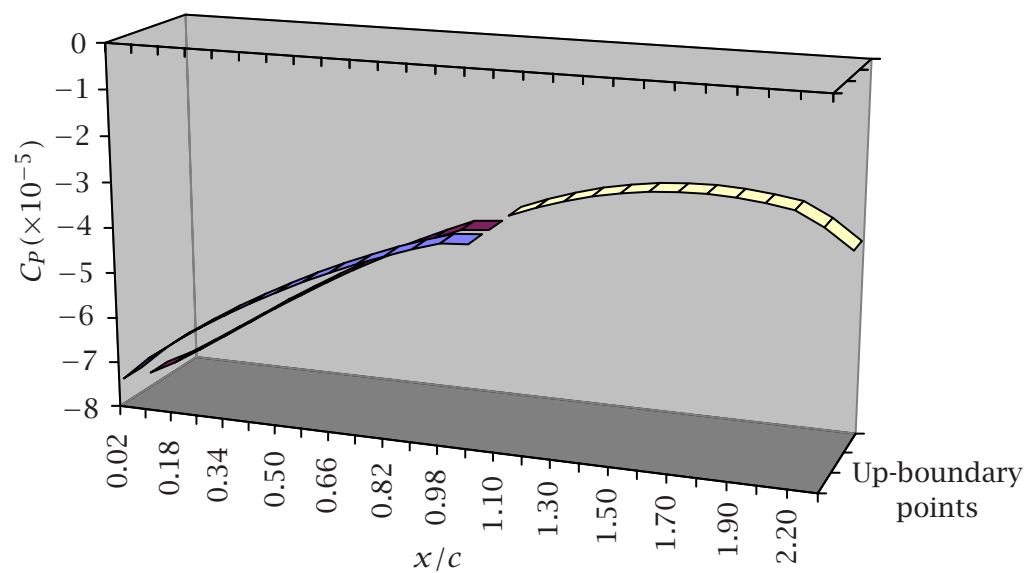

$\square$ Up-boundary points

$\square$ Down-boundary points

$\square$ Out points

FIGURE 6.8. Pressure distribution (3D form) around the aircraft of Figure 5.1, for linear vortex distribution and speed 4 Mach.

From the above figures, it was shown that the values for both up and down points on the boundary of the airfoil are continuously increasing when beginning from $x / c=0$ up to $x / c=1$.

Special attention was given to the investigation of the aerodynamic behaviour of aircrafts, which is currently of continuously increasing interest. The 
special application presented has been used for the determination of the pressure coefficient field around an aircraft airfoil by assuming linear vortex distribution.

7. Conclusions. A nonlinear model has been proposed in the present paper for the determination of the velocity and pressure coefficient field around a NACA airfoil moving by a velocity $U_{A}$ in two-dimensional unsteady flow. Such a problem was reduced to the solution of a nonlinear multidimensional singular integral equation, while this nonlinearity resulted since the source and vortex strength distributions are dependent on their history on the NACA airfoil surface.

On the other hand, closed-form solutions of the nonlinear multidimensional singular integral equations are not possible to be determined, and hence, such type of nonlinear equations has to be solved only by computational methods. In this case some special algorithms must be applied.

Furthermore, a boundary layer model has been proposed in the present paper. Such a model will be ideal for the aerodynamic behaviour of the airfoil as it is able to predict both the laminar and the turbulent parts of the flow as well as the transition region between them. The above boundary layer model was based on the formulation of the unsteady behaviour of the momentum integral equation.

The velocity and pressure coefficient field around an aircraft moving with several velocities was further determined for linear vortex distribution. The proposed method can be applied to the determination of the aerodynamic behaviour of the new generation large aircrafts with very high speeds.

The proposed nonlinear singular integral equation methods will be in the near future of continuously increasing interest for the solution of the generalized solid and fluid mechanics problems. Therefore, special attention should be given to the improvement of singular integral equation methods, as recently many modern problems of fluid mechanics, aerodynamics, solid mechanics, and structural analysis, with big complicated forms, are reduced to nonlinear forms.

\section{REFERENCES}

[1] D. R. Bristow, Development of panel methods for subsonic analysis and design, Tech. Report CR-3234, NASA, USA, 1980.

[2] D. R. Bristow and J. D. Hawk, Subsonic panel method for the efficient analysis of multiple geometry perturbations, Tech. Report CR-3258, NASA, USA, 1982.

[3] F. D. Deffenbaugh and F. J. Marshall, Time development of the flow about an impulsively started cylinder, AIAA J. 14 (1976), 908-913.

[4] R. H. Djojodihardjo and S. E. Widnall, A numerical method for the calculation of nonlinear, unsteady lifting potential flow problems, AIAA J. 7 (1969), 2001-2009.

[5] N. D. Ham, Aerodynamic loading on a two-dimensional airfoil during dynamic stall, AIAA J. 6 (1968), 1927-1934. 
[6] J. L. Hess and A. M. O. Smith, Calculations of non-lifting potential flow about arbitrary three-dimensional bodies, Tech. Report 40622, Douglas Aircraft Company, 1967.

[7] K. Karamcheti, Principles of Ideal-Fluid Aerodynamics, John Wiley \& Sons, New York, 1966.

[8] J. Kim, S. J. Kline, and J. P. Johnston, Investigation of separation and reattachment of a turbulent shear layer: flow over a backward-facing step, J. Fluid Engng. 102 (1982), 392-398.

[9] M. Kiya and M. Arie, A contribution to an inviscid vortex-shedding model for an inclined flat plate in uniform flow, J. Fluid Mech. 82 (1977), 223-240.

[10] E. G. Ladopoulos, Non-linear singular integral computational analysis for unsteady flow problems, Renew. Energy 6 (1995), 901-906.

[11] _ Non-linear singular integral representation for unsteady inviscid flowfields of 2-D airfoils, Mech. Res. Comm. 22 (1995), no. 1, 25-34.

[12] _ Non-linear singular integral representation analysis for inviscid flow fields of unsteady airfoils, Internat. J. Non-Linear Mech. 32 (1997), 377-384.

[13] _ Non-linear multidimensional singular integral equations in twodimensional fluid mechanics, Internat. J. Non-Linear Mech. 35 (2000), no. 4, 701-708.

[14] Singular Integral Equations. Linear and Non-Linear Theory and Its Applications in Science and Engineering, Springer-Verlag, Berlin, 2000.

[15] E. G. Ladopoulos and V. A. Zisis, Existence and uniqueness for non-linear singular integral equations used in fluid mechanics, Appl. Math. 42 (1997), no. 5, 345-367.

[16] _ Nonlinear finite-part singular integral equations arising in twodimensional fluid mechanics, Nonlinear Anal. 42 (2000), no. 2, Ser. A: Theory Methods, 277-290.

[17] H. Lamb, Hydrodynamics, Dover Publications, New York, 1932.

[18] R. I. Lewis, Surface vorticity modeling of separated flows from two-dimensional bluff bodies of arbitrary shape, J. Mech. Engng. Sci. 23 (1981), 1-12.

[19] A. A. Lyrio, J. H. Ferziger, and S. J. Kline, An integral method for the computation of steady and unsteady turbulent boundary layer flows, including the transitory stall regime in diffusers, Tech. Report PD-23, Stanford University, California, 1981.

[20] W. J. McCroskey and S. L. Pucci, Viscous-inviscid interaction on oscillating airfoils in subsonic flow, AIAA J. 20 (1982), 167-174.

[21] J. F. Nash, L. W. Carr, and R. E. Singleton, Unsteady turbulent boundary layers in two-dimensional incompressible flow, AIAA J. 13 (1975), 176-183.

[22] P. E. Robert and G. R. Saaris, Review and evaluation of a three-dimensional lifting potential flow computational method for arbitrary configurations, Boeing Company, 1972.

[23] T. Sarpkaya and H. K. Kline, Impulsively started flow about four types of bluff body, J. Fluid Engng. 104 (1982), 207-213.

[24] T. Sarpkaya and R. L. Schoaff, Inviscid model of two-dimensional vortex shedding by a circular cylinder, AIAA J. 17 (1979), 1193-1200.

[25] R. E. Singleton and J. F. Nash, Method for calculating unsteady turbulent boundary layers in two- and three-dimensional flows, AIAA J. 12 (1974), 590-595.

[26] J. M. Summa, Potential flow about impulsively started rotors, J. Aircraft 13 (1976), 317-319.

E. G. Ladopoulos: Interpaper Research Organization, 56 Anagnostopoulou Street, GR-106 72 Athens, Greece

E-mail address: e1adopou1os@interpaper.org 


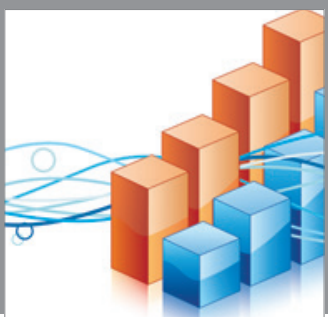

Advances in

Operations Research

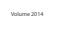

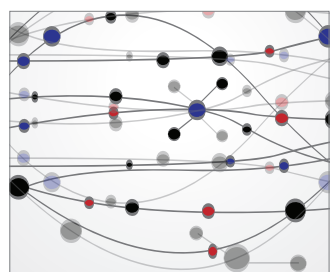

\section{The Scientific} World Journal
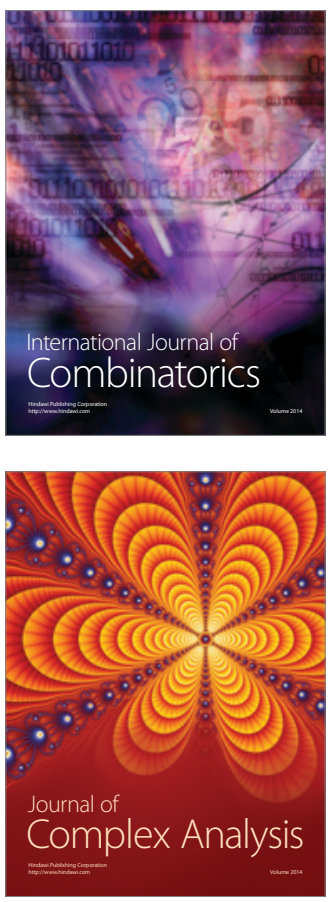

International Journal of

Mathematics and

Mathematical

Sciences
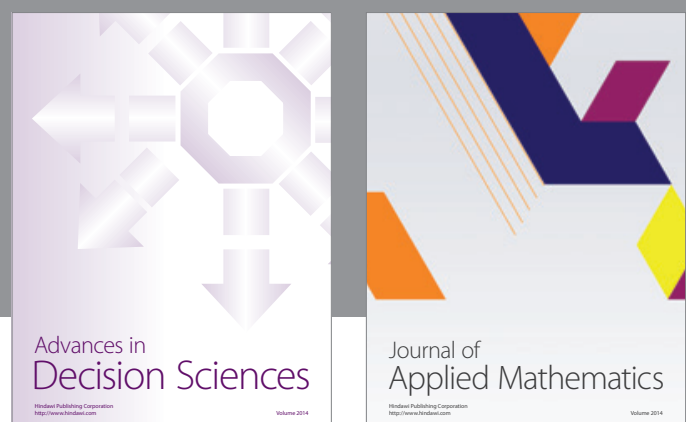

Journal of

Applied Mathematics
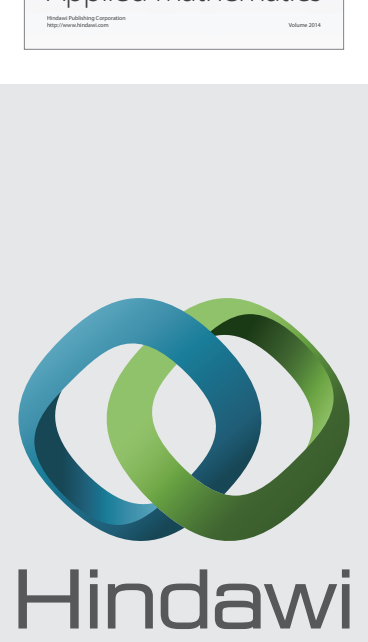

Submit your manuscripts at http://www.hindawi.com
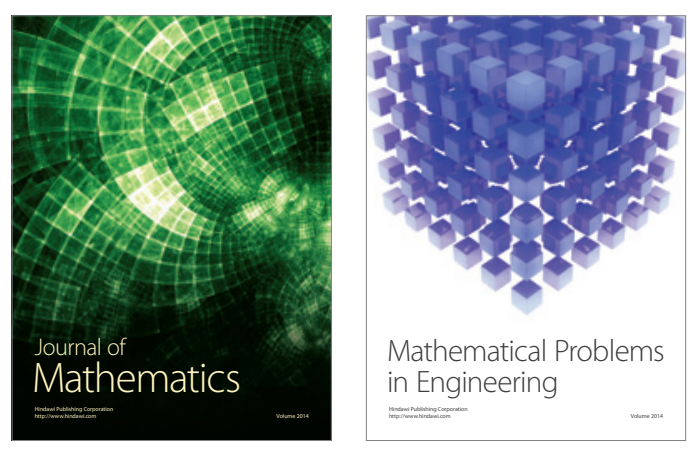

Mathematical Problems in Engineering
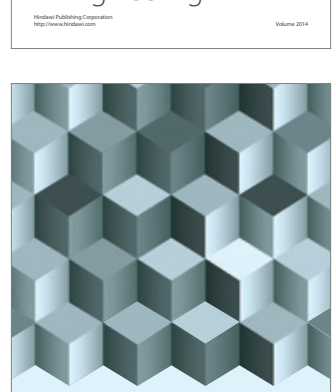

Journal of

Function Spaces
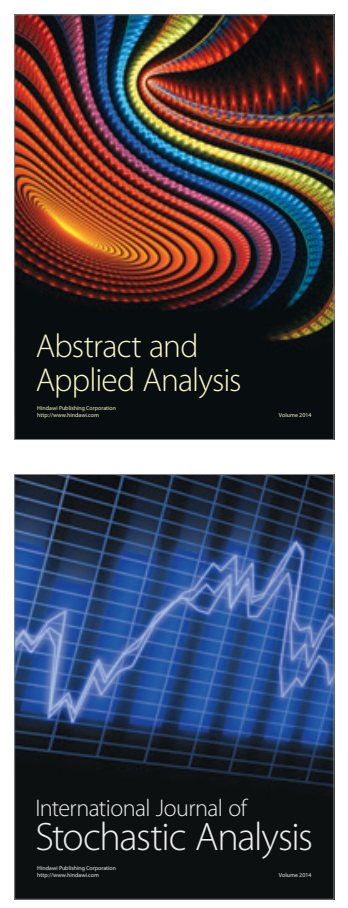

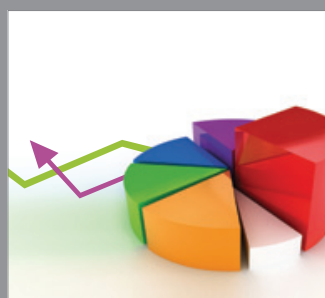

ournal of

Probability and Statistics

Promensencen
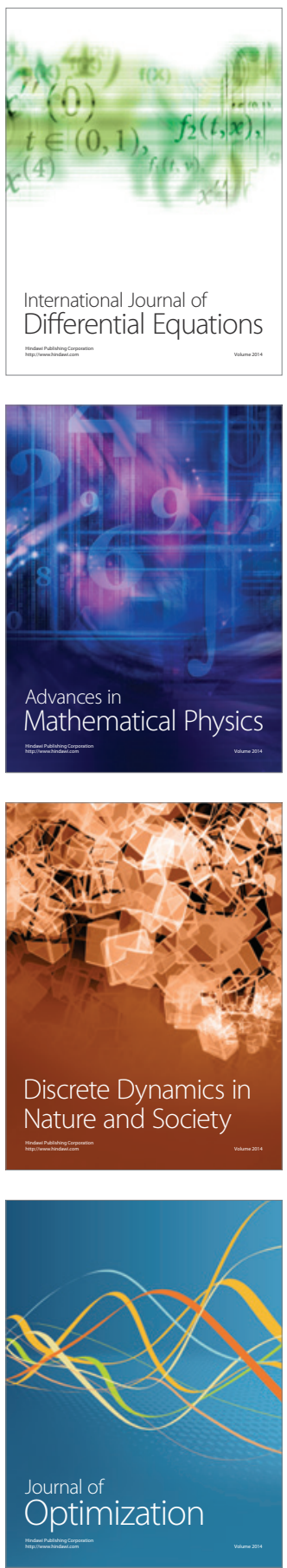